\title{
Right Parasternal Cardiac Surgery after Radical Treatment of Left Breast Cancer
}

Akimasa Morisaki*, Koji Hattori, Yasuyuki Kato, Manabu Motoki, Yosuke Takahashi, Shinsuke Nishimura and Toshihiko Shibata

Department of Cardiovascular Surgery, Osaka City General Hospital, Osaka, Japan

\begin{abstract}
A 74-year-old woman required surgical treatment of severe aortic valve stenosis and two-vessel coronary artery disease consisted of a significant stenosis of the left anterior descending branch and right coronary ostium. She had previously undergone radical mastectomy and radiation therapy for left breast cancer. Severe dermal sclerosis and subcutaneous tissue defect at the chest midline, which would cause poor wound healing because of insufficient blood flow, precluded conventional median sternotomy and a left mini-thoracotomy. Therefore, we selected a hybrid treatment approach using a combination of surgery and percutaneous coronary intervention. We performed aortic valve replacement and coronary bypass grafting to the right coronary artery using a right parasternal approach followed by percutaneous coronary intervention of the left anterior descending artery on postoperative day 13 . Wound healing was quite satisfactory.
\end{abstract}

Keywords: Aortic valve replacement; Breast cancer; Coronary artery bypass grafting; Right parasternal approach; minimally invasive surgery

\section{Introduction}

The use of a right parasternal approach for minimally invasive cardiac surgery has been reported since 1996 [1]. This approach results in a cosmetically acceptable wound and reduced bleeding and infection and, in particular, prevents the disadvantages of median sternotomy [1-4]. We report on a patient who underwent aortic valve replacement and Coronary Artery Bypass Grafting (CABG) via a right parasternal approach. Nearly 30 years earlier, radical treatment of left breast cancer had left the patient with severe dermal sclerosis and an extensive subcutaneous tissue defect; thus, poor wound healing in this area precluded median sternotomy.

\section{Case}

A 74-year-old woman suffered from congestive heart failure with dyspnea on exertion due to severe aortic valve stenosis, for which she was indicated for surgical intervention and referred to our facility. At 46 years of age, she had undergone Halsted radical mastectomy and radiation therapy for left breast cancer. This resulted in a massive subcutaneous tissue defect and a severe dermal sclerosis over the sternum onthe left thorax (Figure 1). Chest X-ray revealed cardiomegaly with protrusion of a left ventricular shadow, slight pulmonary congestion, and a hyper lucent left lung. Echocardiography revealed severe aortic valve stenosis with aortic valve effective orifice area of $0.64 \mathrm{~cm}^{2}$, peak velocity of $4.0 \mathrm{~m} / \mathrm{s}$, and peak pressure gradient of $64 \mathrm{mmHg}$ accompanied by moderate aortic regurgitation. Left ventricular function was maintained, with an ejection fraction of $67 \%$. Additionally, coronary angiography revealed significant stenosis of the left anterior descending branch (\#7) and right coronary ostium (\#1), which would require concomitant surgical treatment. A massive subcutaneous tissue defect of the central and left thorax seen on Computed Tomography (CT) (Figure 2) would prohibit conventional median sternotomy because of poor wound healing due to insufficient blood flow. Thus, we considered a right parasternal approach for aortic valve replacement and only bypass grafting to the right coronary artery. Because the right parasternal approach did not allow access for left CABG, percutaneous coronary intervention of the left anterior descending artery was planned for soon after the operation.

After induction of anesthesia and left double-lumen endotracheal tube ventilation, a Swan-Ganz catheter with a pacing lead was inserted and defibrillation pads were placed on the right and left chest before sterile draping. A $10-\mathrm{cm}$ right parasternal skin incision was made. The right pectoralis major muscle was split at the level of the $3^{\text {rd }}$ and $4^{\text {th }}$ intercostal spaces and a muscle flap created. The right internal thoracic artery was then doubly ligated, and the $3^{\text {rd }}$ and $4^{\text {th }}$ costal cartilages were cut and bent into the left thoracic cavity without removal of the ribs. We also cut the $5^{\text {th }}$ costal cartilage to get better exposure to perform the CABG. After systemic heparinization, cardiopulmonary bypass

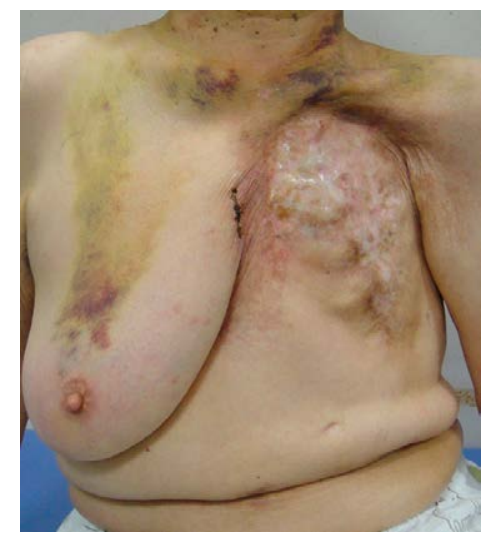

Figure 1: Postoperative photograph shows extensive muscular and subcutaneous tissue defect and dermal sclerosis (broken arrow). The solid arrow shows the location of a right parasternal skin incision of about $10 \mathrm{~cm}$.

*Corresponding author: Akimasa Morisaki, Department of Cardiovascular Surgery, Osaka City General Hospital, 2-13-22 Miyakojima-hondori, Miyakojimaku, Osaka 534-0021, Japan, Tel: +81-6-6929-1221; Fax: +81-6-6929-0623; E-mail: m3_514@yahoo.co.jp

Received December 23, 2013; Accepted February 10, 2014; Published February 16,2014

Citation: Morisaki A, Hattori K, Kato Y, Motoki M, Takahashi Y, et al. (2014) Right Parasternal Cardiac Surgery after Radical Treatment of Left Breast Cancer. J Cardiovasc Dis Diagn 2: 146. doi:10.4172/2329-9517.1000146

Copyright: (c) 2014 Morisaki A, et al. This is an open-access article distributed under the terms of the Creative Commons Attribution License, which permits unrestricted use, distribution, and reproduction in any medium, provided the original author and source are credited. 


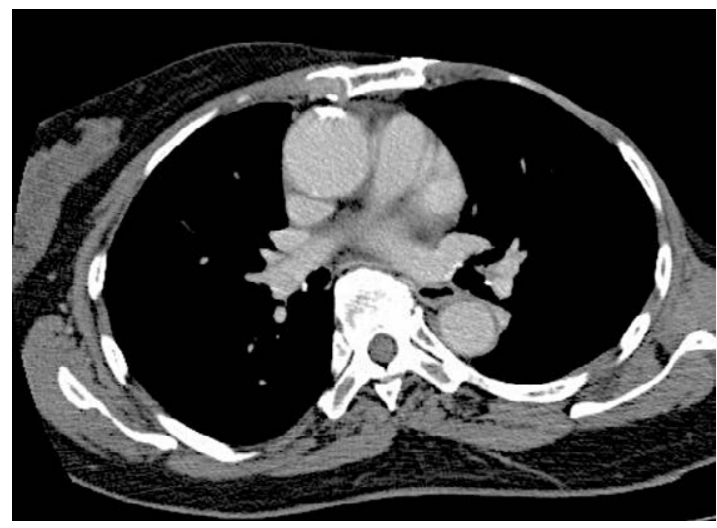

Figure 2: Computed tomography reveals the subcutaneous tissue defect, including left pectoralis major and minor muscle defects, on the left to central thorax (solid arrow).

was established with an 18 -Fr arterial cannula to the right femoral artery and a $25-\mathrm{Fr}$ venous cannula to the right atrium through the right femoral vein. Vacuum-assisted venous drainage was applied. To ensure sufficient venous drainage, we also inserted a venous cannula into the superior vena cava. During cardiopulmonary bypass, a nonpulsatile perfusion flow of over $2.3 \mathrm{~L} / \mathrm{min} / \mathrm{m}^{2}$ was maintained under mild systemic hypothermia. After aortic cross-clamping, identical cold blood cardioplegia was administered first through the aortic root, then intermittently in retrograde fashion. The operative field was flooded with $\mathrm{CO}_{2}$. Through a partial transverse aortotomy, the degenerative aortic valve cups were excised and aortic valve replacement performed, using non-everting mattress sutures to secure a mechanical valve (St. Jude Medical Regent ${ }^{\mathrm{TM}} 17 \mathrm{~mm}$, St. Jude Medical, Inc., Minnetonka, $\mathrm{MN}$, USA). We then performed CABG with a saphenous vein graft to the \#2 branch of the right coronary artery. After uneventful weaning from cardiopulmonary bypass, transesophageal echocardiography showed no paravalvular leakage of the aortic valve. An expanded Polytetrafluoroethylene (PTFE) sheet (GORE ${ }^{\circ}$ PRECLUDE Pericardial Membrane; W.L. Gore \& Associates, Inc., Flagstaff, AZ, USA) was placed under the ribs. After closing the ribs, a continuous-localanesthesia tube was placed under the pectoralis major muscle. The patient was extubated 8 hours after the operation and discharged from the intensive care unit on postoperative day 1 . On postoperative day 13 , she received percutaneous coronary intervention using a drug-eluting stent to the left anterior descending artery, and coronary angiography revealed a patent bypass graft. Postoperative echocardiography showed aortic artificial valve peak velocity of $2.8 \mathrm{~m} / \mathrm{s}$ and peak pressure gradient of $32 \mathrm{mmHg}$ with an ejection fraction of $64 \%$ and no ventricular asynergy. The patient was doing well and discharged without wound complications on postoperative day 16 .

\section{Discussion}

The present case demonstrates that the right parasternal approach facilitated good wound healing in a patient who had undergone radical mastectomy and radiation therapy for left breast cancer.
The right parasternal approach for aortic valve surgery accompanied by CABG to the right coronary artery is an alternative method for patients with valvular and right coronary arterial disease who are not suitable for median sternotomy. Weinschelbaum et al. [2] reported that valve operations with concomitant proximal right CABG could be accomplished through the same incision as a singlevalve operation via a right parasternal approach with cuts to the $3^{\text {rd }}$ and $4^{\text {th }}$ costal cartilages. Our patient, however, required an additional $5^{\text {th }}$ costal cartilage cut for the proximal right CABG because of caudal displacement of the heart with aging. Furthermore, other authors have reported on aortic valve surgery through a right parasternal approach accompanied by left CABG via an additional left mini-thoracotomy in patients requiring concomitant left CABG $[2,3]$. However, in the present case, a left mini-thoracotomy could not be performed because of a severe left thoracic subcutaneous tissue defect resulting from radical treatment for left breast cancer. Therefore, we selected a hybrid treatment approach using a combination of surgery and percutaneous coronary intervention. With this strategy we were able to achieve both an improved prognosis and satisfactory wound healing for this patient.

Disadvantages of the right parasternal approach may include lung hernia and flail chest, which may require surgical treatment and contribute to poor wound healing [4,5]. Cosgrove and Sabik [1] first reported on the parasternal approach for aortic valve replacement with detachment of the $3^{\text {rd }}$ and $4^{\text {th }}$ ribs; however, Cosgrove et al. [4] reported a bulging of the chest wall in the majority of such cases. Minale et al. [3] reported that by avoiding rib resection, the right parasternal approach prevented flail chest. We achieved preservation of the anatomical integrity of the chest wall using the same method by placing an expanded PTFE sheet under the ribs. Preservation of thoracic cage, without the need for rib detachment or reconstruction of the pleura, may prevent these disadvantages.

\section{Conclusion}

We conclude that the right parasternal approach can be used to perform aortic valve replacement and CABG to the right coronary artery when median sternotomy is contraindicated.

\section{References}

1. Cosgrove DM 3rd, Sabik JF (1996) Minimally invasive approach for aortic valve operations. Ann Thorac Surg 62: 596-597.

2. Weinschelbaum $E$, Stutzbach $P$, Machain A, Favaloro $R$, Caramutti $V$, et al. (1998) Valve operations through a minimally invasive approach. Ann Thorac Surg 66: 1106-1109.

3. Minale C, Reifschneider HJ, Schmitz E, Uckmann FP (1998) Minimally invasive aortic valve replacement without sternotomy. Experience with the first 50 cases.Eur J Cardiothorac Surg 14 Suppl 1: S126-129.

4. Cosgrove DM 3rd, Sabik JF, Navia JL (1998) Minimally invasive valve operations. Ann Thorac Surg 65: 1535-1538.

5. Sundara Pandiyan M, Mathew Kavunkal A, Titus VT, Pandarinathan N (2006) Successful repair of chronic instability of anterior chest wall following right parasternal approach for closure of atrial septal defect in a young female. Interact Cardiovasc Thorac Surg 5: 740-741. 